Original Contribution

\title{
BENEFICIAL IMPACT OF HUMAN PLACENTA EXTRACTS ON ERYTHROCYTE MEMBRANE THERMOSTABILITY
}

\author{
O. Nardid, S. Repina*, E. Bobrova, Yu. Govorova, S. Narozhnyi, E. Rozanova \\ Department of Cryobiophysics, Institute for Problems of Cryobiology and Cryomedicine \\ of the National Academy of Sciences of Ukraine, Kharkiv, Ukraine
}

\begin{abstract}
PURPOSE: To study the influence of human placenta extract (HPE) and its individual fractions on the thermal stability of human erythrocyte membrane. METHODS: HPE fractions were isolated by gel chromatography. Thermal hemolysis of erythrocytes, exposed to $55^{\circ} \mathrm{C}$ was measured spectrophotometrically. Cytosol microvscosity and barrier function of erythrocyte membranes at hyperthermia were investigated by EPR spin probe TEMPON. Thermal denaturation of erythrocyte membrane proteins were studied by differential scanning calorimetry. RESULTS: Pre-treatment of erythrocytes with HPE or its fractions inhibited thermal hemolysis. Low-molecular fractions (below 4 $\mathrm{kDa}$ and $12-20 \mathrm{kDa})$ were the most effective in thermal hemolysis inhibition $((31.7 \pm 3.3) \%$ and $(31.5 \pm 3.2) \%$, respectively). The latter fractions markedly reduced the hyperthermia $\left(55^{\circ} \mathrm{C}\right)$-induced permeability of erythrocytes for ferricyanide ions and inhibited the thermo-induced structural transitions in erythrocyte membrane between 40 and $50^{\circ} \mathrm{C}$, which are associated with cytoskeletal proteins. HPE fractions reversibly increased the denaturation temperatures of erythrocyte membrane proteins, except that of spectrin, and enlarged the enthalpy of denaturation of all membrane proteins. CONCLUSIONS: HPE and its individual fractions increased the thermal stability of erythrocyte membranes and erythrocytes. This effect was attributed to the reversible binding of some low molecular ingredient of HPE to the integral proteins and consequent stabilization of their interaction with under-membrane cytoskeleton.
\end{abstract}

Key words: human placenta extracts, human erythrocytes, thermal hemolysis, thermal denaturation of membrane proteins, cytosol microviscosity, membrane barrier function, anti-inflammatory activity.

\section{INTRODUCTION}

The placenta, being the most important organ providing growth and development of the fetus, contains a wide range of biologically active substances that exhibit a number of biological and physiological properties (1). Placenta extracts, obtained by various methods, have been widely used in practical medicine (2) and their therapeutic efficacy is still studied in relation to various pathologies, including inflammatory diseases, osteoarthritis, chronic pain, ischemic brain damage, liver damage, etc. (3-7).

Among the properties of placental extracts researchers especially note their pronounced antiinflammatory activity $(1,8-10)$.

*Correspondence to: Svetlana V. Repina, Institute for Problems of Cryobiology \& Cryomedicine of the National Academy of Sciences of Ukraine Department of Cryobiophysics, 23 Pereyaslavska str., 61016 Kharkiv,Ukraine; e-mail: repina.svetlana@gmail.com
Early studies of Japanese researchers have shown that the anti-inflammatory activity of a number of non-steroidal drugs correlates with their ability to inhibit the thermal hemolysis of erythrocytes (red blood cells, RBCs) $(11,12)$. Based on these and similar reports, the thermal hemolysis of erythrocytes is widely used in testing the potential anti-inflammatory activity of the extracts of various origin (13-15).

It has been shown $(9,10)$ that a pre-treatment of erythrocytes with total HPE inhibited thermal hemolysis, and this process was dose-dependent. These results testify that placenta extract display a stabilizing effect on the erythrocyte membrane. The correlation between the in vitro influence of HPE and its anti-inflammatory activity on living tissues and organisms has been also shown. Up-todate studies are aimed at clarifying which components of HPE exercise this impact (16-18). 
The aim of our research was to study the influence of total aqueous-saline extract of human placenta (HPE) and its individual fractions on the thermal stability of human erythrocytes and proteins of erythrocyte membrane.

\section{MATERIALS AND METHODS}

All studies have been approved by the Bioethics Committee of the Institute for Problems of Cryobiology and Cryomedicine of the National Academy of Sciences of Ukraine and have been performed in accordance with the ethical standards stated in the 1964 'Declaration of Helsinki'. In our study we used total aqueous-saline extract obtained from fresh human placentas as well as its individual fractions, isolated by gel chromatography, as described by us previously (19). Human placentas, weighing between 400$600 \mathrm{~g}$, were collected after normal pregnancies from healthy parturients (23-28 years old Caucasians) with their informed consent. After the full term spontaneous delivery (40 weeks gestation) the placentas were immediately placed under ice. The placentas were not used in case there was an evidence of fetal abnormalities, intrauterine growth restriction, diabetes, hypertension, anemia, tobacco or drug use or other medical or obstetric complications.

The erythrocyte suspensions were prepared from blood obtained from healthy adult volunteers with their informed consent at Kharkiv Regional Center of Blood Service. Erythrocytes of donor blood (male, A (II) +) were isolated from the plasma and white blood cells by a single centrifugation at 1500 $\mathrm{g}$ for $5 \mathrm{~min}$. The next two washings were carried out in an excess of PBS, pH 7.4.

Erythrocyte white ghost membranes were obtained by thrice washing erythrocytes membranes from hemoglobin at $20000 \mathrm{~g}$ for $15 \mathrm{~min}$ in $5 \mathrm{mM}$ sodium phosphate buffer solution, $\mathrm{pH}$ 7.8.

\section{Thermal hemolysis}

For thermal hemolysis studies, the washed RBCs were re-suspended in an equal volume of phosphate-buffered saline (PBS), $\mathrm{pH} \mathrm{7.4,} \mathrm{and} \mathrm{a}$ total HPE or its fractions were added in a ratio of $1: 1$ (v/v). After 1 hour incubation RBCs were washed from HPE and its fractions by centrifugation in excess of PBS at $1500 \mathrm{x} g$ for 5 minutes, re-suspended in PBS and incubated at $55^{\circ} \mathrm{C}$ for 20 minutes in a regulated water thermostat (13). After the incubation, the heated samples were centrifuged at $1500 \mathrm{~g}$ for $5 \mathrm{~min}$ and the hemoglobin content of the supernatant was determined by measuring optical density at $540 \mathrm{~nm}$ by a Pye Unicam SP 8000 spectrophotometer (UK). The hemolysis was calculated as described in (14) and expressed in percents relative to the fully hemolysed cells. The inhibition of hemolysis was determined comparing erythrocytes, preexposed to HPE or its individual fractions with a control sample (erythrocytes pre-exposed in PBS).

\section{Cytosol microviscosity and membrane barrier function at hyperthermia}

The cytosol microviscosity and the barrier function of erythrocyte membranes for small ions (ferricyanide) were investigated at hyperthermia using electron paramagnetic resonance (EPR) spectrometer (Bruker, Rheinstetten, Germany). We used the hydrophilic spin probe TEMPON (SigmaAldrich, St. Louis, USA) in combination with the broadening agent potassium ferricyanide, $\mathrm{K}_{3}[\mathrm{Fe}(\mathrm{CN})]_{6}$, (Sigma-Aldrich, St. Louis, USA), which does not penetrate through intact RBC membranes (Figure 1). This ensured recording EPR signal exclusively from the probes which penetrated into cytoplasm. The EPR spin probe investigations were carried out as described by (19) with some modifications. Briefly, EPR spectra of TEMPON were recorded in the temperature range from 30 to $55^{\circ} \mathrm{C}$ using EPR spectrometer equipped with a thermostat maintaining temperature with accuracy of $\pm 0.5^{\circ} \mathrm{C}$. To determine the rate at which the EPR signal intensity changed, the sample was exposed to $55^{\circ} \mathrm{C}$ in the spectrometer's resonator and the TEMPON spectrum was recorded at each 5 minutes.

EPR spectrum (Figure 1) was used to determine the intensities of the central $\left(h_{0}\right)$ and high-field $\left(h_{-}\right)$ components. $h_{0}$ and the $\left(h_{0} / h_{-}\right)$ratio characterized the ferricyanide permeability of erythrocyte plasma membrane and the microviscosity of cytosol, respectively. The $h_{0} / h_{-}$value as well as its temperature dependence within the range from 30 to $55^{\circ} \mathrm{C}$ characterized the microviscosity of cytosol at hyperthermia. The disturbance of erythrocyte membrane barrier function for ferricyanide ions was evaluated by the decrease in the intensity of the central component $\left(h_{0}\right)$. The time dependence of $\mathrm{h}_{0}$ characterized the rate at which the membrane barrier function was damaged at the temperature of thermal hemolysis.

\section{Thermal denaturation of membrane-bound proteins}

Differential scanning microcalorimetry (DSC) thermograms of erythrocyte ghosts were recorded by a differential adiabatic scanning microcalorimeter (DASM-4, Biopribor, Russia). The probe was heated under 2.5 atm positive pressure from 20 to $95^{\circ} \mathrm{C}$ with heating rate of 1.0 ${ }^{\circ} \mathrm{C} / \mathrm{min}$. The collected data were analyzed using MS Excel and Origin 7.5 (OriginLab Corporation, USA). 


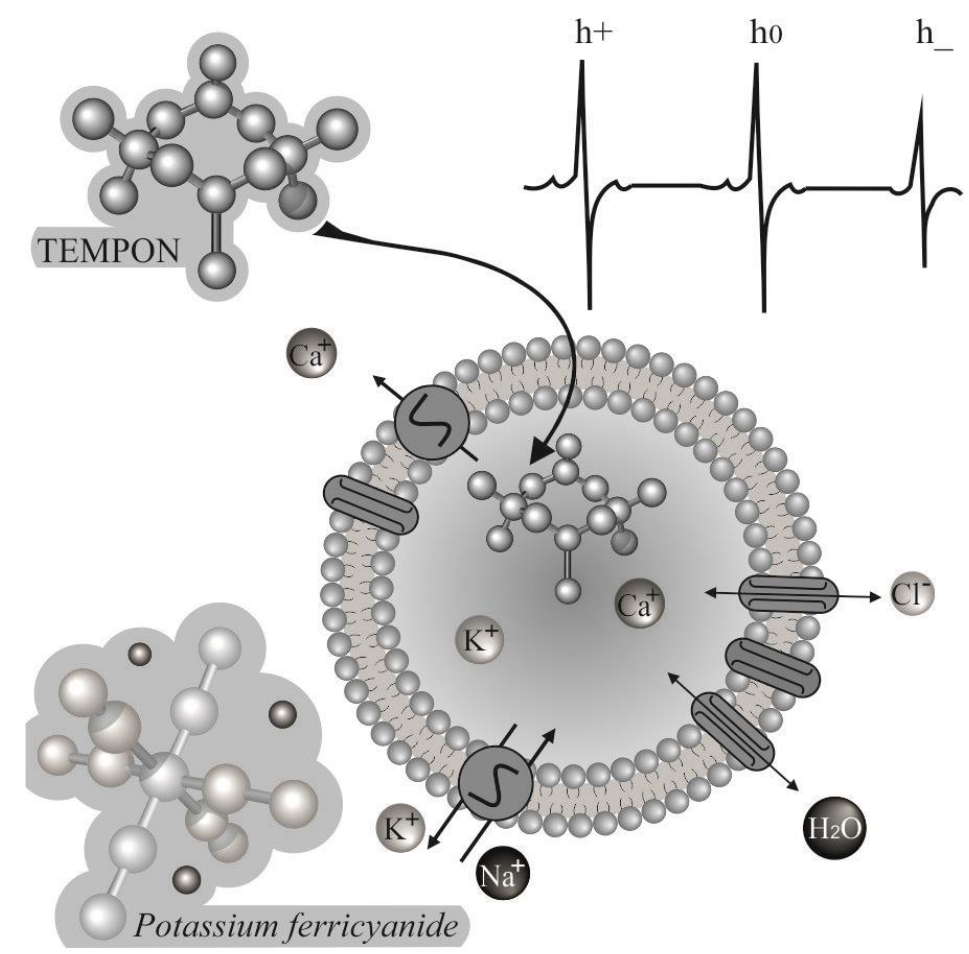

Figure 1. Schematic basics of the EPR method using the TEMPON spin probe with additional broadening of the spin signal by potassium ferricyanide. At the top - spectrum of TEMPON probe placed in the erythrocyte cytosol.

\section{RESULTS AND DISCUSSION}

The hemolysis produced in the suspension of control erythrocytes, incubated at $55^{\circ} \mathrm{C}$ for 20 minutes, was $65.7 \pm 3.9 \%$. Preliminary exposure of erythrocytes to total HPE decreased the level of thermal hemolysis to $53.4 \pm 3.2 \%$ (Table 1).

Table 1. Thermal hemolysis of erythrocytes, pre-treated with total HPE and HPE fractions.

\begin{tabular}{|c|c|c|c|c|c|}
\hline \multicolumn{5}{|c|}{ Hemolysis, \% } \\
\hline \multirow{2}{*}{ Without HPE } & \multirow{2}{*}{ With HPE } & \multicolumn{4}{|c|}{ HPE fractions, kDa } \\
\cline { 3 - 6 } & & $<4$ & $12-20$ & $55-75$ & $>150$ \\
\hline $65.7 \pm 3.9$ & $53.4 \pm 3.2^{*}$ & $44.9 \pm 2.7 *$ & $45 \pm 2.7 *$ & $60.75 \pm 3.7$ & $64.5 \pm 3.9$ \\
\hline
\end{tabular}

* statistically significant difference relative to whole HPE, $p<0.05(n=6)$

Next, we compared the abilities of individual HPE fractions to inhibit hemolysis. Erythrocytes, pre-treated with the low molecular weight fraction $(<4 \mathrm{kDa})$ and the fraction of 12 $20 \mathrm{kDa}$, showed significantly low hemolysis, compared to the whole HPE. Erythrocytes, pretreated with the high-molecular fraction (> 150 $\mathrm{kDa}$ ) showed the highest hemolysis level (Table 1)

In order to better evaluate the ability of different factors to reduce thermal hemolysis, the results are usually expressed as percent inhibition of hemolysis $(13,14)$. For HPE and its fractions the obtained inhibition of hemolysis is shown in Figure 2 in percent. As seen, HPE and all its fractions are able to inhibit hemolysis, but with different power. The ability of the fraction below $4 \mathrm{kDa}$ as well as the fraction 12-20 $\mathrm{kDa}$ was significantly stronger, compared with the total HPE. Fractions with greater molecular weight showed reduced ability to inhibit hemolysis. The percent inhibition of hemolysis by the fractions of $55-75 \mathrm{kDa}$ and that with > $150 \mathrm{kDa}$ was significantly lower in comparison with the total extract (Figure 2).

The thermal hemolysis of erythrocytes has been associated to the increased permeability of erythrocyte membrane at hyperthermia due to a thermo-induced conformational rearrangement of membrane proteins, in particular, a predenaturational rearrangement of band 3 protein (the anion exchanger protein of erythrocyte membrane) $(20,23)$. 


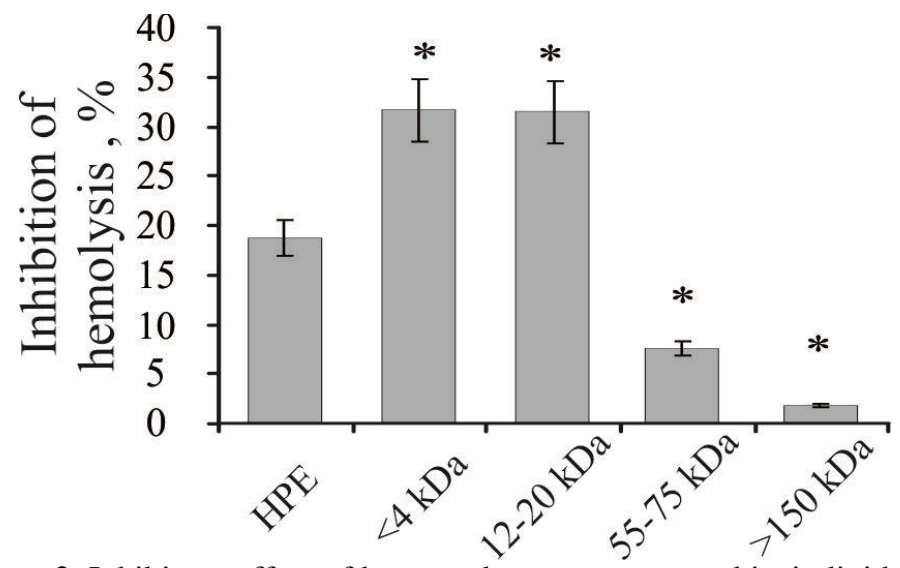

Figure 2. Inhibitory effect of human placenta extract and its individual fractions on the thermal hemolysis of RBCs; * - statistically significant difference in relation to whole HPE, $p<0.05(n=6)$

Several reports have documented that the ability of total HPE to inhibit thermally-induced hemolysis of erythrocytes represents an index of its anti-inflammatory activity $(9,10)$. Inflammation is a complex biological response of the organism and its tissues to harmful factors aiming at protecting itself against these factors and initiating a healing process. In case the inflammation is too strong, a therapy is needed to decrease the intensity of the inflammatory reaction. Inflammation factors cause an increased permeability of cell membranes (13).

The ability of total HPE and its fractions with different molecular weights to reduce thermal hemolysis, as revealed in this study, indicated the ability of these fractions to stabilize the plasma membrane. This can prevent the leakage of proteins and fluids in tissues during the inflammatory process, characterized by increased membrane permeability.

\section{EPR spin probe studies}

The exposure of erythrocytes to $55^{\circ} \mathrm{C}$ was accompanied by a linear decrease in the amplitude of the mid-field $\left(\mathrm{h}_{0}\right)$ component of TEMPON spectrum with the time (Figure 3). This testifies to a disturbance of erythrocyte membrane barrier function and penetration of ferricyanide ions in the cytosole, possibly due to thermo-induced pre-hemolysis alteration of erythrocyte membrane. The slope of the obtained straight line towards the time axis gives an estimate for the induced permeability (rate of membrane barrier function disturbance) and hence, for the erythrocyte membrane stability. Compared to total HPE and other HPE fractions, the HPE fraction with a molecular weight of 12$20 \mathrm{kDa}$ was strongest in decreasing this slope, i.e. in the protection of plasma membranes against thermal damage (Figure 3). As stated above, the same HPE fraction was also the most effective in inhibiting thermal hemolysis.

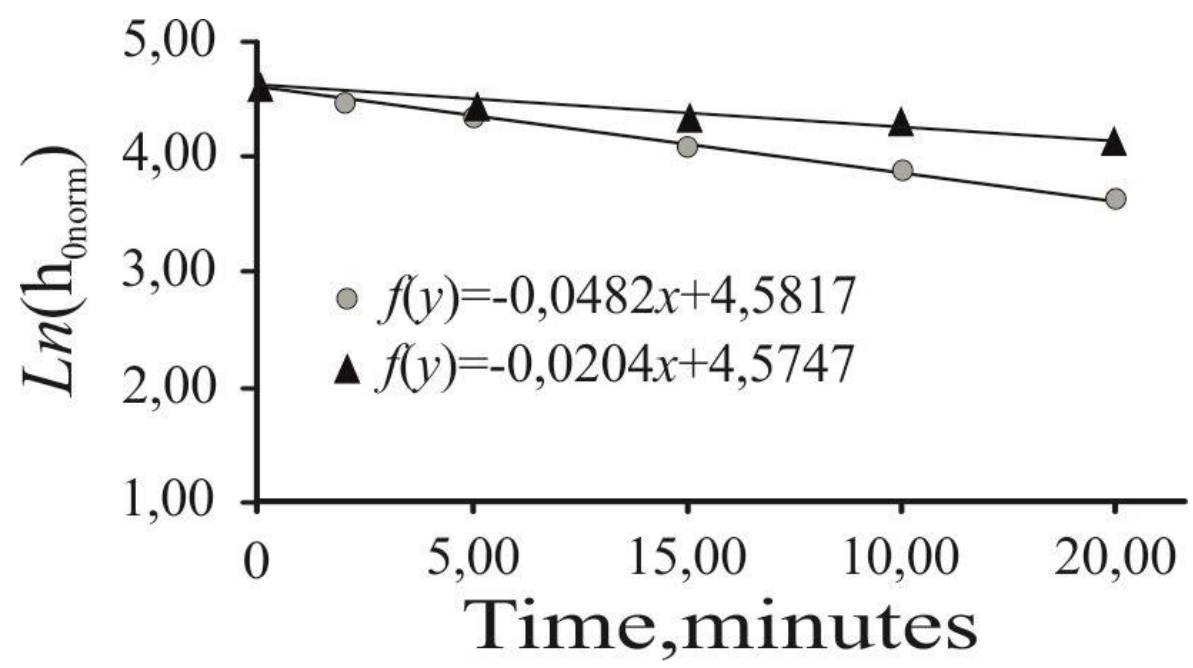

Figure 3. Inhibitory effect of human placenta extract and its individual fractions on the thermally-induced permeability for ferricyanide in erythrocytes exposed to $55^{\circ} \mathrm{C}$. Control erythrocytes - $\circ$; erythrocytes pre-treated with HPE fraction of $12-20 \mathrm{kDa}-\boldsymbol{\Delta}$. The equations represent the linear approximation of experimental data. 
With control erythrocytes, exposed to temperatures between 30 and $55^{\circ} \mathrm{C}$, the temperature dependence of the spectral parameter for cytosole microviscosity, $h_{0} / h_{-}$, showed a thermally-induced structural transition at $40^{\circ} \mathrm{C}$ (Figure 4). This transition has been associated with the thermotropic rearrangement
NARDID O., et al. of the main cytoskeleton protein, spectrin (21). Also, this temperature dependence curve demonstrated pronounced changes in its slope at $46-47^{\circ} \mathrm{C}$ (the initial stage of spectrin denaturation (22) and at $50^{\circ} \mathrm{C}$ (the denaturation temperature of spectrin (23).

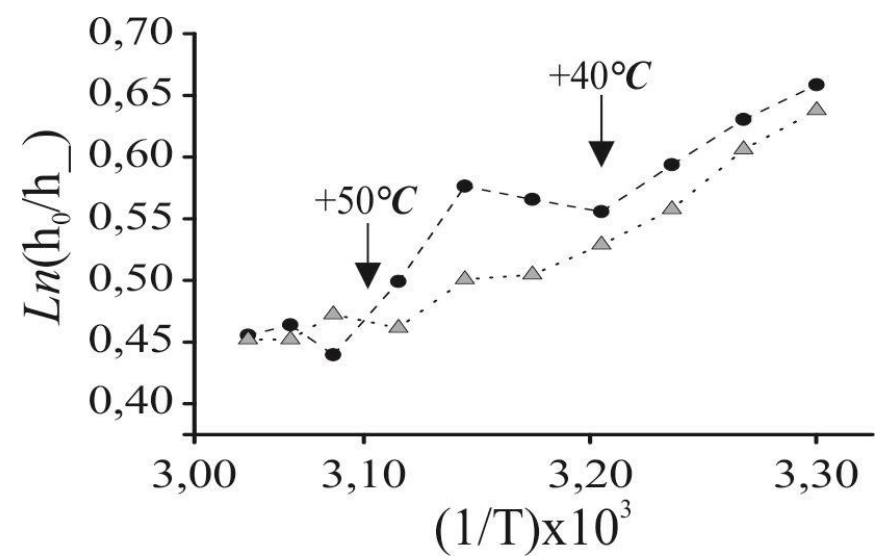

Figure 4. Effect of HPE-treatment of erythrocytes on the Arrhenius dependence of the microviscosity parameter of TEMPON in erythrocyte cytosol: Control erythrocytes - • erythrocytes pre-treated with total HPE -

The pre-treatment of erythrocytes with HPE modified the temperature dependence curve of the TEMPON microviscosity parameter (Figure 4). Strong smoothing of this curve (inhibition of thermotropic rearrangements) and a decrease in cytosol microviscosity took place, thereat only the change in the region of $40-50^{\circ} \mathrm{C}$ remained. This result also evidenced for the beneficial impact of HPE on erythrocyte membrane.

\section{Thermal denaturations of membrane-bound proteins}

The beneficial impact of HPE on the erythrocyte membranes was further studied by DSC analysis of the thermal denaturations of RBC membrane proteins as affected by the high-, medium- and low-molecular fractions of HPE.
In line with previous reports $(20,24)$, the DSC detected four major endothermic peaks, corresponding to different protein denaturations (structural transitions) in erythrocyte membrane. They took place at the temperatures $51 \pm 0.1^{\circ} \mathrm{C}$, $58 \pm 0.1^{\circ} \mathrm{C}, 67 \pm 0.1^{\circ} \mathrm{C}$ and $80 \pm 0.1^{\circ} \mathrm{C}$, commonly denoted as $\mathrm{A}, \mathrm{B}, \mathrm{C}$ and $\mathrm{D}$ peaks (transitions), respectively (Figure 5, curve 1). There are several classifications of these denaturation transitions (24). According to Shnyrov (25), these transitions implicate the thermal denaturations of the following proteins, A transition - spectrin (electrophoretic bands 1 and 2) and ankyrin (band 2.1); transition B - bands 4.2, 4.9 proteins and actin (band 5); transition $\mathrm{C}$ - transmembrane domain of band 3, band 4.1 and glyceraldehyde phosphate dehydrogenase (band 6); transition D - tropomyosin (band 7).

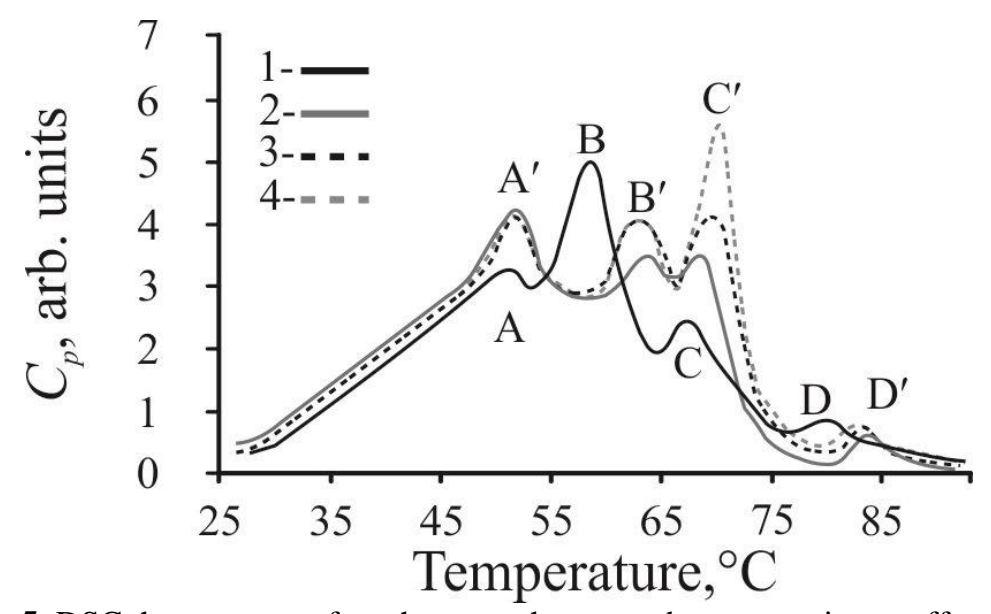

Figure 5. DSC thermogram of erythrocyte ghost membrane proteins as affected by HPE extracts: control membranes -1 ; membranes with fraction $\langle 4 \mathrm{kDa}-2$; membranes with fraction $>$ $150 \mathrm{kDa}-3$; membranes with fraction of $50-60 \mathrm{kDa}-4$. 
Two-hour exposure of erythrocyte ghost membranes to HPE fractions with different molecular weights increased the top temperature and/or the area of the denaturation peaks. Regardless of their molecular weight, all fractions increased the top temperatures of B, C, and $\mathrm{D}$ denaturation peaks (Table 2). An exception was the A peak whose temperature, regardless of the molecular weight of the fraction, remained within the margins of error. At the same time, each HPE fraction
NARDID O., et al. significantly increased the area of A peak (Figure 5, peak $\mathbf{A}^{\prime}$ ). The increase in the area of other peaks (B', C', D') depended on the molecular weight of the fraction. The increase in the denaturation temperature (thermal stability) and peak area (denaturation enthalpy) both evidenced for strong structural stabilization of erythrocyte membrane proteins under the impact of total HPE and its low molecular fractions.

Table 2. Denaturation temperatures of RBC membrane proteins as affected by the HPE fractions.

\begin{tabular}{|c|l|l|l|l|}
\hline $\begin{array}{l}\text { MW of Peak } \\
\text { the HPE fraction, kDa }\end{array}$ & A & B & C & D \\
\hline without HPE fraction & $51 \pm 0.1$ & $58 \pm 0.1$ & $67 \pm 0.1$ & $80 \pm 0.1$ \\
\hline$>150$ & $51 \pm 0.1$ & $62 \pm 0.1^{*}$ & $70 \pm 0.1^{*}$ & $82 \pm 0.1^{*}$ \\
\hline $50-60$ & $51 \pm 0.1$ & $62 \pm 0.1^{*}$ & $71 \pm 0.1^{*}$ & $82 \pm 0.1^{*}$ \\
\hline$<4$ & $51 \pm 0.1$ & $63 \pm 0.1^{*}$ & $69 \pm 0.1^{*}$ & $83 \pm 0.1^{*}$ \\
\hline
\end{tabular}

$*-\mathrm{p}<0.05$ relative to erythrocyte ghost membranes without HPE fractions

This increase in the area and top temperature of the denaturation peaks indicated a reversible or irreversible binding of some active components of HPE fractions to the proteins of erythrocyte membranes. To check the reversibility of the binding, prior to DSC test the HPE-treated RBC ghosts were washed out of the HPE fraction components using sodium phosphate buffer. Figure 6 compares the DSC thermograms of intact (untreated) RBC ghost membranes (Figure 6, curve 1), and of membranes subjected to two-hour exposure to the (strongest) fraction with lowest molecular weight $(<4 \mathrm{kDa})$ and subsequently washed out prior to the DSC test (Figure 6, curve 2). The two thermograms were almost identical indicating that the binding of biologically active components of HPE was reversible one.

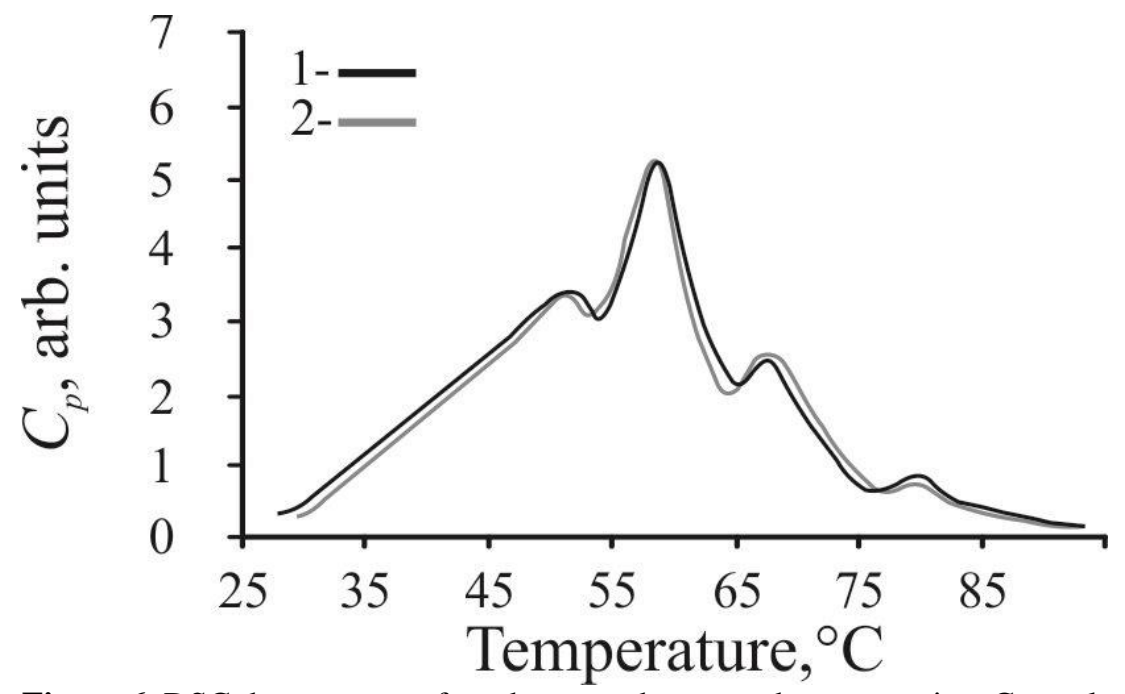

Figure 6. DSC thermogram of erythrocyte ghost membrane proteins. Control membranes - 1; membranes pre-treated and washed with a HPE fraction $<4 \mathrm{kDa}-2$.

Thus, we assume the pretreatment the HPE fractions modified erythrocyte membrane integral proteins, predominantly band 3, increasing their structural stability. Because the integral proteins are tightly anchored to several peripheral proteins of under-membrane cytoskeleton (band 2.1; band 4.1; actin), the latter proteins became also stabilized (peak B'). By contrast, fibrilar protein spectrin is not directly bound to the integral proteins and was only partially stabilized by area (peak A'). Hence, the stabilizing effect of HPE and its 
fractions, in particular the low molecular weight (<4 kDa) fraction, could involve not only reversible binding to outside aspect of integral proteins but also an effect on the "weak" interactions in the under-membrane cytoskeletal cortex.

\section{CONCLUSION}

Our results demonstrate the beneficial impact of aqueous-saline human placenta extract on the thermal and structural stability of human erythrocyte membrane. Human placenta extracts with different molecular weight were obtained and their inhibiting effect on thermal hemolysis and stabilizing effect on membrane proteins was documented. The fractions with lowest molecular weight (below $4 \mathrm{kDa}$ and 12-20 kDa) were the most effective. Most probably some substances of later fractions stipulate the antiinflammatory activity of human placental extract.

\section{ABBREVIATION}

HPE - human placenta extract, EPR - electron paramagnetic resonance, DSC - differential scanning calorimetry, RBC - red blood cell, PBS - phosphate-buffered saline.

\section{REFERENCES}

1. Pan, SY, Chan, M. K.S., Wong, M. B. F., Klokol, D. and Chernykh, V. Placental therapy: An insight to their biological and therapeutic properties. J Med Therap, 1(3): 16, 2017

2. Chakraborty, PD., Bhattacharyya, D. Aqueous Extract of Human Placenta as a Therapeutic Agent. In: Recent Advances in Research on the Human Placenta. Rijeka,Croatia: InTech pp 77-92, 2012.

3. Prameela, Sharma, KD Clinical efficacy of placentrex injection in pelvic inflammatory disease. Indian Journal of Obstetrics and Gynaecology Research, 3(1):65-67, 2016.

4. Park, KM, Cho, TH. Therapeutic effect of acupuncture point injection with placental extract in knee osteoarthritis. J Integr Med., 15(2):135-141, 2017.

5. Marleau, AM., McDonald, G., Koropatnick, J., Chen, CS., Koos, D. Reduction of tumorigenicity by placental extracts. Anticancer Res; 32:1153-1161, 2012.

6. Park, JY, Byeon, JH, Park, SW, Eun, SH, Chae, KY, Eun, BL. Neuroprotective effect of human placental extract on hypoxicischemic brain injury in neonatal rats. Brain Dev, 35(1):68-74, 2013.

7. Jung, J., Lee, HJ., Lee, JM, Na, KH, Hwang, SG, Kim, GJ. Placenta extract promote liver regeneration in CCl4-injured liver rat model. Int Immunopharmacol, 11:976-84, 2011.

8. Kawakatsu, M., Urata, Y., Goto, S., Ono, Y., and $\mathrm{Li}, \mathrm{TS}$. Placental extract protects bone marrow-derived stem/progenitor cells against radiation injury through anti-inflammatory activity. J Radiat Res, 54(2): 268-276, 2013.

9. Banerjee, K.K., Bishayee, A. and Chatterjee, M. Role of human placental extract on succinic dehydrogenase activity in carrageenin-induced edema in rats in vivo and its effect on erythrocyte lysis, platelet aggregation and trypsin activity in vitro. Indian J Physiol Pharmacol, 38(2): 121-124, 1994.

10.Banerjee, K.K., Bishayee, A. and Chatterjee, M. Anti-inflammatory effect of human placental extract: a biochemical mechanistic approach. Riv. Eur. Sci. Med. Farmacol, 14(6): 361-366, 1992.

11.Mizushima, Y., Sakai, S., Yamaura, M. Mode of stabilizing action of non-steroid anti-inflammatory drugs on erythrocyte membrane. Biochemical Pharmacology, 19(1): 227-234, 1970.

12.Mizushima Y., Ishii, Y., and Masumoto, S. Physico-chemical properties of potent nonsteroidal anti-inflammatory drugs Biochemical Pharmacology, 24(17): 15891592, 1975,

13.Anosike, Ch.A., Obidoa, On. and Ezeanyika, L. Membrane stabilization as a mechanism of the anti-inflammatory activity of methanol extract of garden egg (Solanum aethiopicum). DARU Journal of Pharmaceutical Sciences, 20:76, 2012. doi: 10.1186/2008-2231-20-76.

14.Bhadoriya SS., Mishra V., Raut S., Ganeshpurkar, A. and Jain, SK. AntiInflammatory and antinociceptive activities of a hydroethanolic extract of tamarindus indica leaves. Sci Pharm, 80(3): 685-700, 2012.

15.Bougandoura, A., D'Abrosca, B., Ameddah, S., Scognamiglio, M., Mekkiou, R., Fiorentino, A., Benayache, S., Benayache, F. Chemical constituents and in vitro antiinflammatory activity of Cistanche violacea Desf. (Orobanchaceae) extract Fitoterapia, 109(2): 248-253, 2016.

16.Sharma, K. and Bhattacharyya, D. Immunoglobulin isotype isolated from human placental extract does not interfere in complement-mediated bacterial opsonization within the wound milieu. FEBS Open Bio, 5: 369-377, 2015.

17.De, D., Chakraborty, PD., Bhattacharyya, D. Analysis of free and bound NADPH in aqueous extract of human placenta used as wound healer. $J$ Chromatogr B Analyt Technol Biomed Life Sci, 877 (24): 24352442, 2009.

18.De, D., Chakraborty, P.D., Bhattacharyya, D. Regulation of trypsin activity by peptide fraction of aqueous extract of human placenta 
used as wound healer. J Cell Physiol. 226 (8): 2033-2040, 2011.

19.Nardid, O., Repina, S., Rozanova, E., Cherkashina, Ya., Nardid, E. Properties of aqueous-saline human placental extracts and their fractions after storage of placenta at various subzero temperatures. J Exp Integr Med, 5(4): 172-177, 2015.

20.Ivanov, I.T., Brähler, M., Georgieva, R., Bäumler, $\mathrm{H}$. Role of membrane proteins in thermal damage and necrosis of red blood cells. Thermochimica Acta, 456: 7-12, 2007.

21.Minetti M., Ceccarini M., Di Stasi A. M. et al. Spectrin involvement in a 40 degrees $\mathrm{C}$ structural transition of the red blood cell membrane // J Cell Biochem. - 1986. - Vol. 30, № 4. - P. 361-370.

22.Iamaǐkina I.V., Mansurov V.A., Ivashkevich E.V. Temperature denaturation of erythrocyte
NARDID O., et al. spectrin: rheology, deformability and resistance to detergents // Biofizika. - 1997. Vol. 42, № 3. - P. 675-679.

23.Ivanov I.T., Zheleva A., Zlatanov I. Anion exchanger and the resistance against thermal haemolysis // Int. J. Hyperthermia. - 2011. Vol. 27, №3. - P. 286-296.

24.Matveev A.V., Akoev V.R., Tarakhnovskii Yu.S. Deev A.A., Bryukhanov V.M., Zhadan G.G. \& Shnyrov V.L. (1997). A comparative study of structural transitions in erythrocyte membranes of adult donors and neonates. Bull. Exp. Biol. Med., 123, No 2, pp. 196200.

25.Lapshina E.I. \& Zavodnik I.B. (1994). Thermostability of erythrocyte membrains proteins with erythrocyte and medium compounds varying. Biophizika, Vol. 39, No 6, pp. 1015-1020. 
NARDID O., et al. 